\title{
The Benefits and Burdens of Cancer: A Prospective Longitudinal Cohort Study of Adolescents and Young Adults
}

\author{
Joelle P. Straehla, MD, ,2 Krysta S. Barton, PhD, MPH, ${ }^{3,4}$ Joyce P. Yi-Frazier, PhD, \\ Claire Wharton, BS, ${ }^{3}$ Kevin Scott Baker, MD, MS, ${ }^{3,5,6}$ Kira Bona, MD, MPH, ${ }^{1,2,7,8}$ \\ Joanne Wolfe, MD, MPH, ${ }^{1,7-9}$ and Abby R. Rosenberg, MD, MS ${ }^{3-6}$
}

\begin{abstract}
Background: Adolescents and early young adults (AYAs) with cancer are at high risk for poor outcomes. Positive psychological responses such as benefit-finding may buffer the negative impacts of cancer but are poorly understood in this population.

Objective: We aimed to prospectively describe the content and trajectory of benefit- and burden-finding among AYAs to develop potential targets for future intervention.

Patients and Methods: One-on-one semistructured interviews were conducted with English-speaking AYA patients (aged 14-25 years) within 60 days of diagnosis of a noncentral nervous system malignancy requiring chemotherapy, 6-12 and 12-18 months later. Interviews were coded using directed content analyses with $a$ priori schema defined by existing theoretical frameworks, including changed sense of self, relationships, philosophy of life, and physical well-being. We compared the content, raw counts, and ratios of benefit-toburden by patient and by time point.

Setting/subjects: Seventeen participants at one tertiary academic medical center (mean age 17.1 years, $\mathrm{SD}=2.7)$ with sarcoma $(n=8)$, acute leukemia $(n=6)$, and lymphoma $(n=3)$ completed 44 interviews with $>100$ hours of transcript-data.

Results: Average benefit counts were higher than average burden counts at each time point; $68 \%$ of interviews had a benefit-to-burden ratio $>1$. Positive changed sense-of-self was the most common benefit across all time points (44\% of all reported benefits); reports of physical distress were the most common burden (32\%). Longitudinal analyses suggested perceptions evolved; participants tended to focus less on physical manifestations and more on personal strengths and life purpose.

Conclusions: AYAs with cancer identify more benefits than burdens throughout cancer treatment and demonstrate rapid maturation of perspectives. These findings not only inform communication practices with AYAs but also suggest opportunities for interventions to potentially improve outcomes.
\end{abstract}

Keywords: adolescent and young adult; benefit-finding; communication; palliative care; pediatric cancer; psychosocial

\footnotetext{
${ }^{1}$ Department of Pediatric Hematology/Oncology, Harvard Medical School, Boston, Massachusetts.

${ }^{2}$ Department of Pediatric Oncology, Dana-Farber Cancer Institute, Boston, Massachusetts.

${ }^{3}$ Cancer and Blood Disorders Center, Seattle Children's Hospital, Seattle, Washington.

${ }^{4}$ Treuman Katz Center for Pediatric Bioethics, Seattle Children's Hospital, Seattle, Washington.

${ }^{5}$ Department of Pediatrics, University of Washington, Seattle, Washington.

${ }^{6}$ Clinical Research Division, Fred Hutchinson Cancer Research Center, Seattle, Washington.

${ }^{7}$ Department of Medicine, Boston Children's Hospital, Boston, Massachusetts.

${ }^{8}$ Division of Population Sciences, Dana-Farber Cancer Institute, Boston, Massachusetts.

${ }^{9}$ Department of Psychosocial Oncology and Palliative Care, Dana-Farber Cancer Institute, Boston, Massachusetts.

Accepted December 1, 2016.
} 


\section{Introduction}

$\mathbf{T}$ HE EXPERIENCE OF cancer among adolescents and early young adults (AYAs) is unique because it may interfere with normal developmental transitions. ${ }^{1,2}$ Perhaps for these reasons, AYAs have not experienced the same improvements in survival ${ }^{3-5}$ and have disparate psychosocial outcomes compared to younger pediatric and older adult patients. ${ }^{6-12}$

Targeted interventions to improve these outcomes are necessary, but several challenges must be overcome to develop them effectively. First, there are few longitudinal descriptions of AYA patient distress, ${ }^{13}$ coping, or adjustment mechanisms; much of the literature is cross sectional, retrospective, and/or nongeneralizeable. ${ }^{14}$ Second, studies tend to emphasize negative outcomes such as post-traumatic stress or distress. ${ }^{9,15}$ A concurrent focus on positive psychosocial outcomes may be informative.

Positive outcomes of cancer have been described, including constructs such as post-traumatic growth ${ }^{16}$ and benefitfinding. ${ }^{17}$ These related phenomena are common following a range of traumatic events such as natural disasters, ${ }^{18}$ chronic illnesses, ${ }^{19,20}$ and cancer. ${ }^{21-23}$ When studied longitudinally, these constructs appear protective; they are associated with lower levels of depression, anxiety, pain, and functional disability. ${ }^{24}$ Taken together, positive psychological outcomes like benefit-finding may buffer the impact of trauma-related distress. ${ }^{20}$ This is particularly important among AYAs who have a high potential for positive psychological adaptation after having had cancer. ${ }^{25,26}$

To our knowledge, benefit-finding has not been studied in a prospective manner among AYAs with cancer. To develop potential targets for future intervention, we aimed to longitudinally characterize concurrent AYA patient-reported benefit- and burden-finding during the first one to two years of the cancer experience.

\section{Patients and Methods}

\section{Participants}

The "Resilience in Adolescents and Young Adults" study was a multicenter, prospective, longitudinal mixed-methods study investigating contributors and inhibitors of resilience. ${ }^{27}$ The present subanalysis describes findings of serial, semistructured 1:1 interviews conducted at a single site. Eligible participants were 14-25 years of age and had been diagnosed with a noncentral nervous system (CNS) malignancy requiring multiagent systemic chemotherapy within the 60 days before enrollment. Those with CNS tumors were excluded to limit heterogeneity related to cognitive impairment. All consecutive eligible participants diagnosed between December 2012 and July 2013 were approached; written informed consent (ages 18 and older) or assent with parental consent (ages 14-17) was obtained in accordance with the local Institutional Review Board.

\section{Qualitative interviews}

Interviews were conducted by a single trained investigator (A.R.R.) with consecutive newly enrolled participants until subsequent interviews suggested no new themes describing patient-reported resilience. ${ }^{27,28}$ Participants were interviewed 1:1 (without parents present) at the time of their cancer
TABle 1. Interview Guide

Opening question

"How has this [cancer experience] been for you?"

Exploratory items

Participant expectations, beliefs, worries, and concerns about cancer

Participant reported personal strengths and challenges

Sources of support

Coping strategies

Quality of life

Closing questions

"How do you define resilience?" or, "What does

'resilience' mean to you?"

"Knowing what you know now, what advice would you give a [same-aged] patient with [same diagnosis] who was diagnosed today?',

diagnosis, 6-12 and 12-18 months later. The interview location was determined pragmatically based upon participants' treatment schedules and included the following: (1) private interview rooms in the institution's clinical research center when participants were outpatient and (2) private hospital rooms when participants were hospitalized. Interviews lasted between 30 and 90 minutes each and included open-ended questions eliciting expectations, beliefs, and worries about cancer, plus participant-identified strengths and challenges (Table 1).

\section{Qualitative analyses}

Verbatim transcripts were coded by three independent coders (J.P.S., K.S.B., A.R.R.) for all instances of benefit- or burden-finding, defined as participant-identified positive or negative effects or outcomes resulting from their cancer experience. Directed content analysis ${ }^{28}$ was used to develop an initial coding scheme; this method was used to leverage existing theoretical frameworks to measure psychosocial outcomes after trauma. Three major a priori coding themes were derived from validated scales (the Post-traumatic Growth Inventory ${ }^{16}$ and the Benefit and Burden Finding Scale for Children $^{29}$ ): changed sense of self, changed sense of relationships, and changed philosophy of life. Within each theme, coders identified both positive comments (i.e., benefit-finding) as well as negative comments (i.e., burdenfinding). Following deductive analyses of all interviews conducted at the time of enrollment, the same coders created one additional major code to capture physical well-being based on the data and mutual consensus. Several additional subthemes were then developed to classify all coded instances of benefit- and burden-finding identified in the interviews (Supplementary Table S1; Supplementary Data are available online at www.liebertpub.com/jpm). All interviews (including those from the time of enrollment) were then reanalyzed. Discrepancies were cataloged and discussed until group consensus was reached; overall agreement was $95 \%$.

Theme 1: changed sense of self. Post-traumatic growth theory suggests a traumatic event such as cancer not only causes distress but also increases the sense that one is being tested and "surviving"; thus, it can spark both negative and positive changes in participants' views of themselves. ${ }^{16}$ 
Positive (benefit-finding) changes include becoming a stronger person, developing new coping skills, and appreciating "the small things" in life. Negative changes (burdenfinding) include diminished confidence, increased anxiety, low mood, and less enjoyment of life (Supplementary Table S1).

Theme 2: changed sense of relationships. The experience of personal vulnerability often prompts the need for reliance on close relationships, ${ }^{16}$ with both positive and negative implications. Changes in personal relationships may relate to family dynamics and/or friendships. Relationships with romantic partners were included under friendships as not all participants disclosed or were in romantic relationships. Positive changes include growing closer to others, new or greater appreciation of family, development of new friendships, and identification of authentic friendships. Negative changes include worry about burdening others emotionally or financially, having less time for friendships, and being treated differently (Supplementary Table S1).

Theme 3: changed philosophy of life. This theme is driven by existential questions about life's purpose, which can prompt positive or negative changes in participants' relationship to the world at large. Changes include those regarding religion or spirituality as well as societal views. Coding schema for religion or spirituality included both affective (connection to a source beyond oneself) and cognitive (behavioral or religious practices) domains. ${ }^{30}$ Positive changes include finding meaning in the diagnosis or developing a stronger faith; negative changes included lost or decreased faith. Changes in societal views were coded as positive when participants identified, for example, new priorities in life and the desire to help others; codes for negative changes include feeling disengaged from society and having to witness the suffering of others (Supplementary Table S1).

Theme 4: physical well-being. Participants identified beneficial as well as burdensome changes in their physical well-being related to their cancer diagnosis, prompting the addition of this theme. Positive (beneficial) changes include increased understanding of personal health and acknowledgement of the therapeutic value of cancer-related interventions (e.g., chemotherapy, radiation, supplemental nutrition through nasogastric tube) on their recovery. Negative (burdensome) changes included side effects of treatment, as well as concerns of disability, infertility, and other late effects (Supplementary Table S1).

\section{Quantitative analyses}

Raw counts of benefit- and burden-finding were calculated for each participant interview with ratios (defined separately as benefit:burden per participant and per population) and $95 \%$ confidence intervals (CIs). To better understand the natural history of benefit- and burden-finding, the raw and proportional counts within each theme and subtheme were compared at each time point. Although all interviews were included in raw and proportional assessments by time points, comparative longitudinal analysis included only those participants who completed all three interviews.

\section{Results}

\section{Participant characteristics}

Seventeen participants completed the first interview, and 12 completed all 3 interviews. Their ages ranged from 14 to 23 years at diagnosis (average 17.1 \pm 2.7 ) (Table 2).

Eight $(47 \%)$ were male, and the most common diagnoses were sarcoma $(n=8)$, acute leukemia $(n=6)$, and lymphoma $(n=3)$. The majority of the participants $(82 \%)$ were nonHispanic White.

Time between first interview (T1) and second interview (T2) averaged 3.5 \pm 1 months, and time between T2 and the third interview (T3) averaged $12 \pm 3.5$ months. Reasons for attrition included progressive disease $(N=2)$, death $(N=1)$, relocation $(N=1)$, and declined further participation $(N=1) .{ }^{31}$ Interviews totaled $>100$ hours and transcripts totaled 324 single-spaced pages.

\section{Comparison of benefit- and burden-finding}

Each participant interview had multiple benefit and burden counts with variable numbers of each in any given interview (benefit median 15, range 9-79; burden median 16, range 454 per interview). At each time point, the average benefit counts per interview were higher than average burden counts (Fig. 1A).

In the majority of interviews ( 33 of $44,68 \%$ ), the ratio of benefit count to burden count was $>1$ (Fig. 1B); however, there was notable interparticipant variability at each time point and each participant's trajectory over time was unique. For example, while some participants had linear patterns of increasing benefit-to-burden ratios over time, other participants had decreasing ratios or nonlinear sporadic patterns.

\section{Analysis by theme}

Changed sense of self. Positive changes regarding sense of self were the most commonly reported benefits overall (44\% of all reported benefits [95\% CI 37-52], Table 3). Nearly all participants remarked on a new or

Table 2. Characteristics of Patients Interviewed AT Time Point $1(N=17)$

\begin{tabular}{lc}
\hline & Mean $($ SD $)$ \\
\hline Age at diagnosis (years) & $17.1(2.7)$ \\
Sex, $n(\%)$ & $8(47)$ \\
Male & $9(53)$ \\
Female & \\
Diagnosis, $n(\%)$ & $8(47)$ \\
Sarcoma & $4(24)$ \\
ALL & $2(12)$ \\
Hodgkin lymphoma & $2(12)$ \\
AML & $1(6)$ \\
NHL & \\
Race, $n(\%)$ & $14(82)$ \\
Non-Hispanic White & $1(6)$ \\
Hispanic & $1(6)$ \\
Other & $1(6)$ \\
Refused & \\
\hline
\end{tabular}

ALL, acute lymphoblastic leukemia; AML, acute myeloid leukemia; NHL, non-Hodgkin lymphoma; SD, standard deviation. 

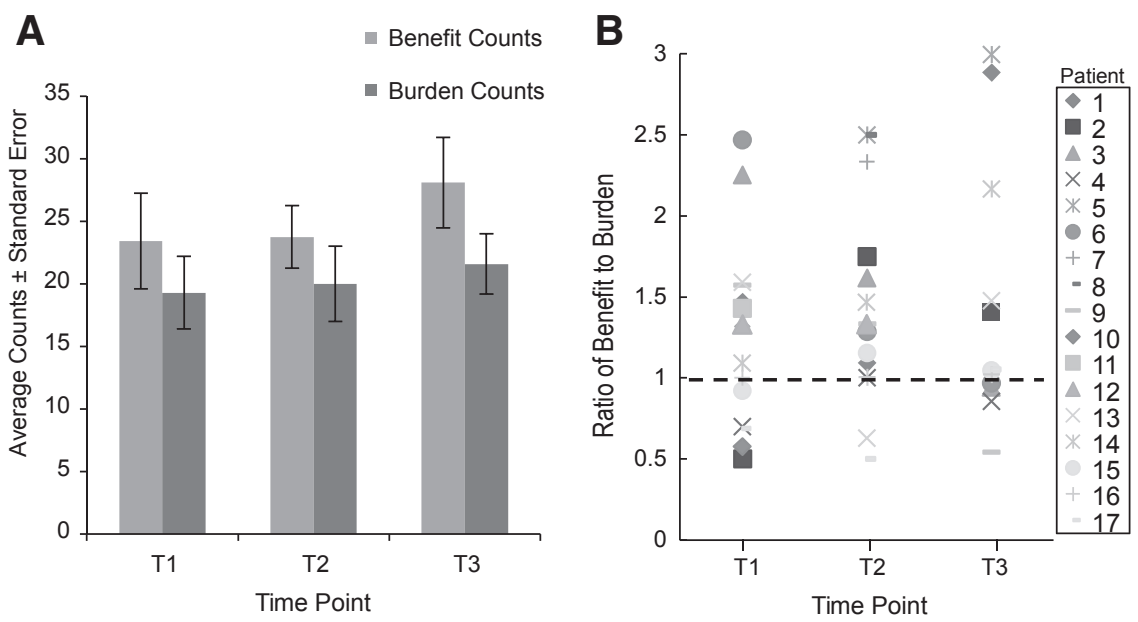

FIG. 1. (A) Average counts of benefit-finding (positive comments) and burden-finding (negative comments) per interview with AYA participant at each of three time points (T1, T2, T3). The error bars represent standard error. (B) The ratio of counts of benefit-finding to counts of burden-finding (benefit-to-burden ratio) plotted separately for participant interviews at each of the three time points (T1, T2, T3). Each patient is represented by a unique symbol as indicated. The horizontal dashed line represents a ratio of 1 such that participant interviews plotted above the line had higher benefit counts than burden counts. AYA, adolescent and early young adult.

expanded sense of personal strength ("people always said that I was strong, but I never really knew how strong I was"). Personal coping mechanisms were commonly identified, including positive thinking, goal setting, and reframing challenges, as well concrete practices for stress management. Many participants also described personal growth in areas such as patience, empathy, and new appreciation for things already present in their lives ("[I] value the simple things. Like, feeling good enough to eat out and hang out with your friends compared to before I just took all of it for granted").

Burdens related to changed sense of self were also frequently identified, encompassing $25 \%$ of all burdens $(95 \%$ CI 19-31). Many participants endorsed periods of feeling down or depressed ("I would just feel sad or feel hopeless or whatever... it's wearing me down and I'm just sick and tired of being sick and tired"), general feelings of anxiety ("worry it's, like, a daily thing-it's just worry all the time"), or having overall less enjoyment in life ("I'm a little more negative than like I used to be"). Particularly common were sentiments of insecurity or lack of confidence related to appearance. For example, hair loss and other changes in appearance due to treatment were important sources of distress.

Changed sense of relationships. Overall, changed relationships encompassed $25 \%$ of all benefits (95\% CI 1832 , Table 2); of these, comments about family comprised $40 \%$ ("family, they'll always be there...they're still the main support") and friends $60 \%$ ("I would've never thought I would've developed these incredible and, like, rich relationships with these people until—as sad as it is—till I got sick") (Table 3).

Burdens regarding relationships encompassed $26 \%$ of all burdens (95\% CI 19-32, Table 2); of these, family made up $35 \%$ and friends made up $65 \%$. Participants commonly identified guilt or worry over burdening those close to them by their diagnosis ("I'd hurt a lot of people by being diagnosed. People were crying, people were sad") or the rigors of treatment ("it's a lot for them to go through... my mom has to leave my little sister and come here with me and I feel-I feel bad"). Nearly all participants expressed difficulty communicating with friends or distress over being treated differently by friends because of their diagnosis ("[my] friends were just freaking out and I had a few not speak to me because they just didn't know how to handle it at first...people prepared themselves for my death").

Changed philosophy of life. Changed philosophy of life encompassed $22 \%$ of all benefits (95\% CI 14-30, Table 3); of these, $32 \%$ were religious/spiritual and $68 \%$ were societal. Commonly identified religious or spiritual benefits included a sense of meaning or purpose ("I think that it's just God did it for a reason to make me stronger") and feeling supported by a religious community ("when someone's praying about me... the pain in cancer goes away."). Changed societal views included having a new outlook on life and a desire to help others.

Burdens regarding changed philosophy of life were the least commonly identified, encompassing $17 \%$ of all burdens (of these, $70 \%$ were religious/spiritual, $30 \%$ were societal $[95 \%$ CI 11-24], Table 3). Religious or spiritual burdens predominantly encompassed existential distress and fear of death or relapse ("I watched my grandpa die of [cancer]...so I'm kind of nervous sometimes, like, I'm-I'm too young...I don'tI'm not ready"). While societal burdens were the least common overall, several participants identified feeling disengaged or viewing the world as a more negative or dark place ("seeing like five year olds and six year olds and like infants going in for the stuff that I'm struggling with...it takes its toll”).

Physical well-being. Physical well-being made up the smallest percentage of all identified benefits (8\% [95\% CI 611], Table 3). These included improved health knowledge 


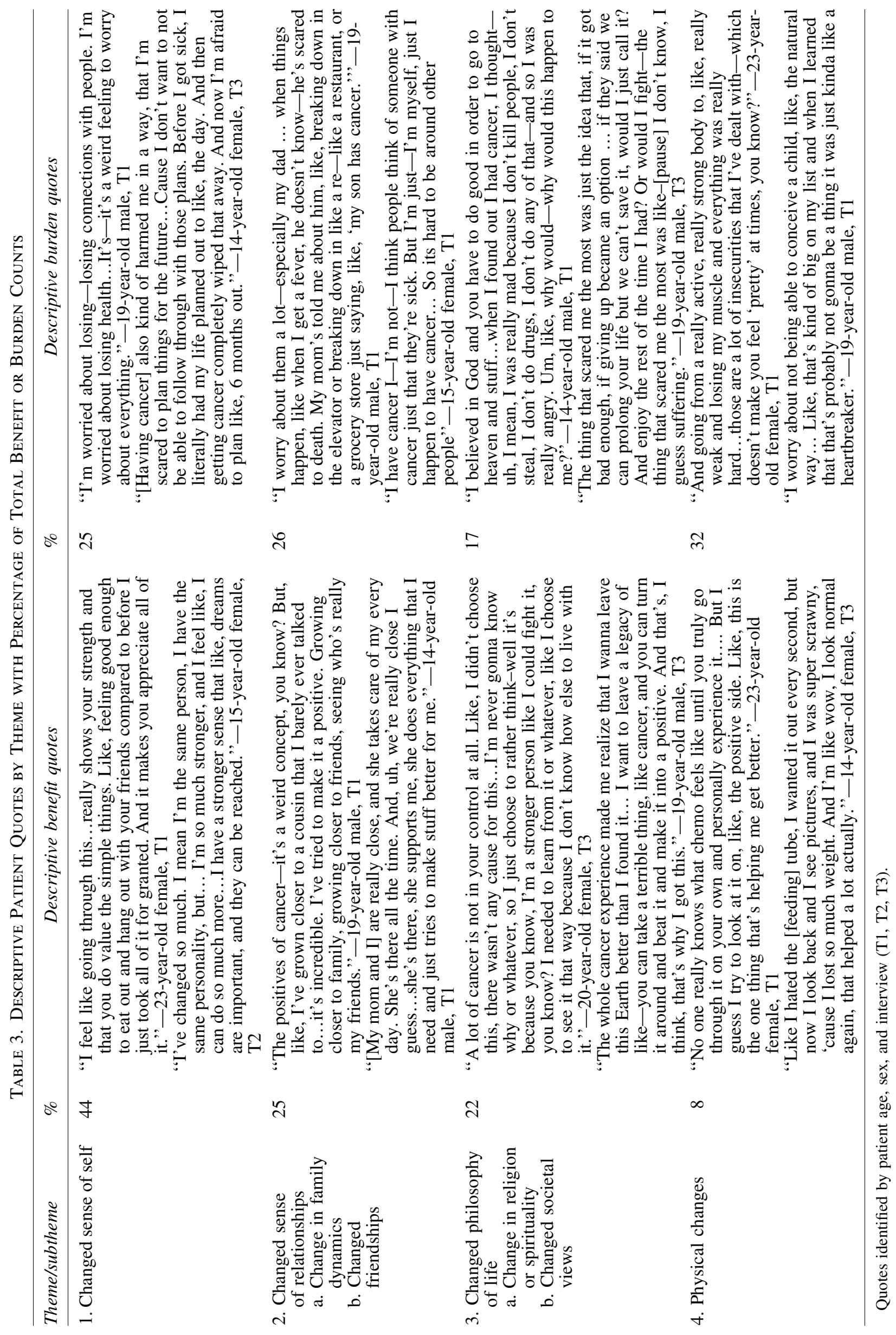




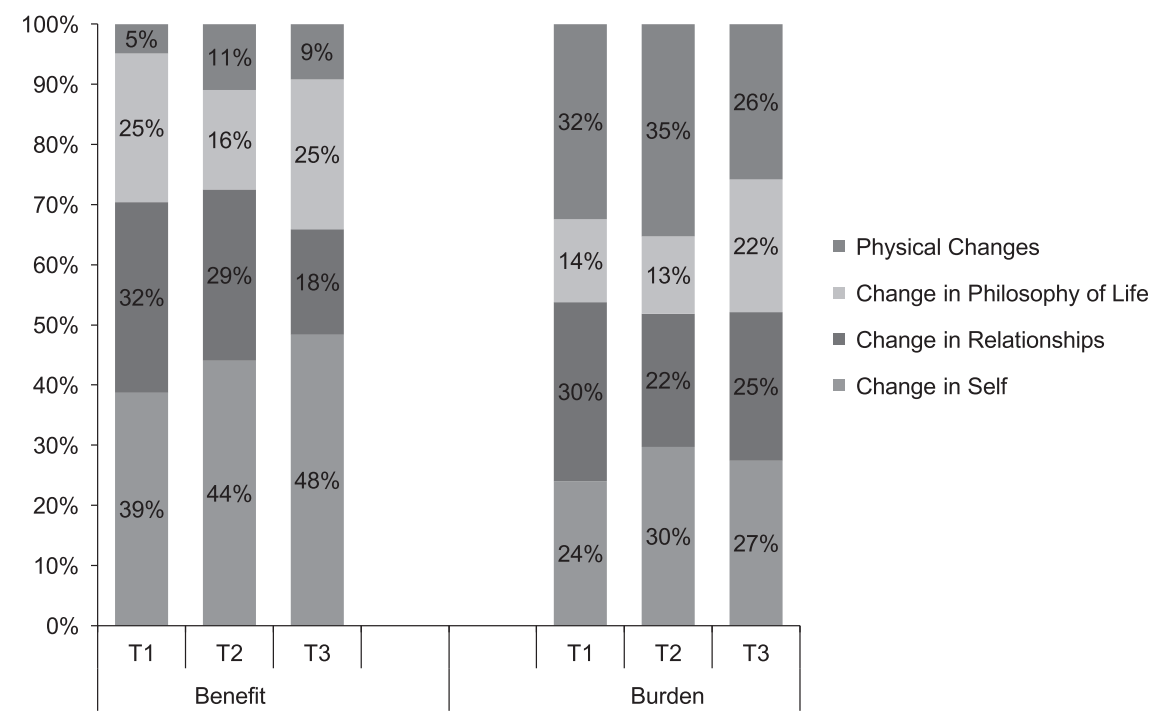

FIG. 2. Benefit and burden counts at each interview time point (T1, T2, T3) were normalized to 100\%, and the percentage breakdown by theme (change in self, change in relationships, change in philosophy of life, and physical changes) is shown over time for benefit and burden, respectively. The data shown incorporate participants who completed all three interviews $(N=12)$.

and acknowledgment that therapies were beneficial to their recovery ("it's been nice... watching my leg...respond to the chemo."). In contrast, reports of physical distress were the most common burden identified overall (32\% [95\% CI 2539]), with the majority of participants identifying physical distress related to receiving chemotherapy, surgery, and radiation ("just the first time getting [chemotherapy] - that's the hardest thing I've ever done-in my seventeen years"). Less concrete burdens were also commonly identified, including the threat of disability, new illness, intimacy issues, and infertility.

\section{Longitudinal analysis}

Among the 12 participants completing all 3 interviews, findings suggested an evolution of patient-identified benefits and burdens. Specifically, comments reflected increasing identification of benefit related to changed sense of self and less identification of benefits related to changed sense of relationships over time (Fig. 2). Identification of physical complaints remained a large proportion of the burden counts but decreased over time. There was an increase in burdens related to change in philosophy of life.

Qualitatively there were three common shifts noted over time: a more positive view of self, an increase in existential awareness, and a new more positive outlook on life (Table 4).

\section{Discussion}

In this prospective longitudinal analysis of AYA patientreported benefits and burdens of cancer, we found that benefit- and burden-finding exist concurrently. Participants

Table 4. Descriptive Patient Quotes at Interview 1 (T1) and Interview 3 (T3) Showing Change in Benefit- and Burden-Finding Over Time

\begin{tabular}{|c|c|c|}
\hline & Patient at $T 1$ & Same patient at T3 \\
\hline More positive view of self & $\begin{array}{l}\text { "There's, like, days where I just don't } \\
\text { feel very strong...I have an image, } \\
\text { like, of crossing the finish line but } \\
\text { there are some days when I just can't } \\
\text { picture that image." }\end{array}$ & $\begin{array}{l}\text { "I see myself as like, this new } \\
\text { person...I've become more, um, } \\
\text { adventurous, and not afraid to live in } \\
\text { the moment...Because you never } \\
\text { know how long you're gonna have } \\
\text { to do that." }\end{array}$ \\
\hline Increased existential awareness & $\begin{array}{l}\text { "[I think about why this happened] a } \\
\text { little bit but I can never come to any } \\
\text { conclusions... Just I don't really } \\
\text { know. I don't think it's terribly } \\
\text { important-the why...I don't know } \\
\text { if there's even a reason.". }\end{array}$ & $\begin{array}{l}\text { "I've been in a situation where I've } \\
\text { been told, 'oh it's possible you could } \\
\text { die next year'... having to face that } \\
\text { reality, changed my perspective } \\
\text { 'cause that could happen again.", }\end{array}$ \\
\hline New, more positive outlook on life & $\begin{array}{l}\text { "I know going through [cancer] } \\
\text { changes people and I'll have a } \\
\text { different perspective. But I'm } \\
\text { afraid...I'll be so different that my } \\
\text { friends won't want me anymore." }\end{array}$ & $\begin{array}{l}\text { "[It] was a really difficult thing to go } \\
\text { through... definitely the most } \\
\text { horrible time of my life, but it } \\
\text { wasn't overall bad ...I'm like a } \\
\text { better, happier person because I've } \\
\text { like really suffered now." }\end{array}$ \\
\hline
\end{tabular}


tended to identify more benefits than burdens at the time of diagnosis and during the two years thereafter. Furthermore, their comments suggest a shift in perspectives over time, including a progressively more positive view of self and an evolving more positive outlook on life.

These findings provide important initial descriptions of the natural history of benefit-finding in the AYA population with cancer. Indeed, how AYAs identify and articulate benefit-finding has not previously been well described. While we found that many constructs from prior theories applied to AYAs (e.g., changes in self, relationships, and philosophy of life), the content and details of these constructs within the AYA population are, not surprisingly, unique. For example, adolescence and young adulthood are characterized by developmental changes in individuality, including the establishment of independence, social circles, sexual identity, and sense of self. ${ }^{2,32}$ For participants in our study, these processes were manifest in their comments about both benefits and burdens of cancer. They recognized (and were burdened by) their lack of independence. They learned to appreciate authentic and dependable friendships. In addition, perhaps because of their age-appropriate focus on body image and physical identity, these AYAs also commented frequently on the physical benefits and burdens of cancer and its requisite treatment. Therefore, physical benefit- and burden-finding may be an important and understudied construct. Finally, while it has been theorized that stressful events such as illness can halt adolescent neurodevelopment or even cause regression, ${ }^{33-35}$ patients in our study provided multiple examples of maturity and evolving wisdom; many created meaning and life purpose from their experiences.

This wisdom beyond their years is not uncommon among AYAs with cancer and may, in part, be related to changed perspectives (e.g., from invincibility toward existential awareness) after a life-threatening cancer diagnosis. In a study comparing AYA cancer survivors and age-matched well peers given hypothetical end-of-life scenarios, for example, survivors were more likely to consider palliative options, even it if meant that the hypothetical patient would die sooner. ${ }^{36}$ Similarly, AYAs with advanced cancer understand early on that they are no longer invincible and that their experience will profoundly impact their loved ones. ${ }^{37}$ The vast majority express not only a willingness but also a readiness to discuss complex issues such as advanced care plans and locations of death. ${ }^{38,39}$ Taken together, these data advance the understanding of AYA emotional maturation in the context of serious illness.

The presence of benefit-finding among AYAs with cancer is important because it is a hallmark of positive psychological outcomes $^{16,17}$ and may predict more functional coping and adaptation over time. ${ }^{24}$ In this high-risk population, this is relevant for both patients who survive their cancers and those who do not. Targeted interventions promoting benefit-finding may improve immediate coping, health, and psychosocial outcomes during survivorship, as well as aid in advance care planning during the experience of progressive or incurable cancer. Future research should focus on investigating the association between benefit-finding and improved long-term outcomes among AYAs with cancer, interventions to promote benefit-finding, and strategies to address some modifiable sources of burden-finding unique to AYAs such as physical identity perceptions, intimacy issues, and infertility.
There are several limitations to this study. Participants were enrolled from a single urban center with limited ethnic diversity and, while representative of the region, may not be generalizable to other populations. Attrition over time may impact our longitudinal analysis as those with progressive disease or death could not be included. We did not track location of interviews, although this may have influenced patient perceptions; inpatients may have focused more on immediate physical symptoms. In addition, we may have inadvertently promoted benefit-finding simply by inviting participants to share their story, as therapeutic narratives have been shown to enable coping and patient engagement. ${ }^{31,40}$ Given the exploratory nature of the work, a control group could not be utilized. Furthermore, with the aim of characterizing AYA patient-reported benefit- and burden-finding, data came from coded interviews as opposed to quantitative measures, such that there are no standardized scores to compare our findings to other patient populations. However, studying benefit- and burden-finding in this way (with likefor-like themes) allowed for other, novel quantitative comparisons within this cohort, adding a new perspective to the AYA arc of experiences.

The presence and content of AYA patient-reported benefitfinding both soon after diagnosis and during treatment are encouraging and underscore a potential opportunity for targeted interventions designed to promote positive outcomes while buffering negative ones. Ultimately, research in this arena may improve health and psychosocial outcomes in this high-risk population.

\section{Acknowledgments}

St. Baldrick's Fellow award; Young Investigator award from CureSearch for Children's Cancer; Clinical Research Scholar Award from Seattle Children's Hospital's Center for Clinical and Translational Research; National Center for Advancing Translational Sciences of the National Institutes of Health; Grant number: KL2TR000421

An abstract from this work was presented in poster form at the ASCO Palliative Care Symposium (September 9-10, 2016) and an oral presentation is scheduled at the American Academy of Hospice and Palliative Medicine Meeting (February 22-25, 2017).

\section{Author Disclosure Statement}

No competing financial interests exist.

\section{References}

1. D’Agostino NM, Penney A, Zebrack B: Providing developmentally appropriate psychosocial care to adolescent and young adult cancer survivors. Cancer 2011;117:23292334.

2. Zebrack BJ: Psychological, social, and behavioral issues for young adults with cancer. Cancer 2011;117:2289-2294.

3. Bleyer A: Young adult oncology: The patients and their survival challenges. CA Cancer J Clin 2007;57:242-255.

4. Tai E, Pollack LA, Townsend J, et al.: Differences in nonHodgkin lymphoma survival between young adults and children. Arch Pediatr Adolesc Med 2010;164:218-224.

5. Thomas DM, Seymour JF, O'Brien T, et al.: Adolescent and young adult cancer: A revolution in evolution?. Intern Med J 2006;36:302-307. 
6. Evan EE, Zeltzer LK: Psychosocial dimensions of cancer in adolescents and young adults. Cancer 2006;107:1663-1671.

7. Kazak AE, Barakat LP, Meeske K, et al.: Posttraumatic stress, family functioning, and social support in survivors of childhood leukemia and their mothers and fathers. J Consult Clin Psychol 1997;65:120-129.

8. Phillips-Salimi CR, Lommel K, Andrykowski MA: Physical and mental health status and health behaviors of childhood cancer survivors: Findings from the 2009 BRFSS survey. Pediatr Blood Cancer 2012;58:964-970.

9. Sansom-Daly UM, Wakefield CE: Distress and adjustment among adolescents and young adults with cancer: An empirical and conceptual review. Transl Pediatr 2013;2:167-197.

10. Schultz KA, Ness KK, Whitton J, et al.: Behavioral and social outcomes in adolescent survivors of childhood cancer: A report from the childhood cancer survivor study. $\mathbf{J}$ Clin Oncol 2007;25:3649-3656.

11. Smith AW, Bellizzi KM, Keegan TH, et al.: Health-related quality of life of adolescent and young adult patients with cancer in the United States: The Adolescent and Young Adult Health Outcomes and Patient Experience study. J Clin Oncol 2013;31:2136-2145.

12. Stuber ML, Meeske KA, Krull KR, et al.: Prevalence and predictors of posttraumatic stress disorder in adult survivors of childhood cancer. Pediatrics 2010;125:e1124-e1134.

13. Kwak M, Zebrack BJ, Meeske KA, et al.: Trajectories of psychological distress in adolescent and young adult patients with cancer: A 1-year longitudinal study. J Clin Oncol 2013;31:2160-2166.

14. O'Leary TE, Diller L, Recklitis CJ: The effects of response bias on self-reported quality of life among childhood cancer survivors. Qual Life Res 2007;16:1211-1220.

15. Phipps S, Klosky JL, Long A, et al.: Posttraumatic stress and psychological growth in children with cancer: Has the traumatic impact of cancer been overestimated?. J Clin Oncol 2014;32:641-646.

16. Tedeschi RG, Calhoun LG: The Posttraumatic Growth Inventory: Measuring the positive legacy of trauma. J Trauma Stress 1996;9:455-471.

17. Calhoun LG, Tedeschi RG: The foundations of posttraumatic growth: An expanded framework. In: RG Tedeschi and LG Calhoun (eds.), Handbook of Posttraumatic Growth: Research and Practice, 1st ed. Lawrence Erlbaum and Associates: New York, NY, 2006, p. 1.

18. McMillen JC, Smith EM, Fisher RH: Perceived benefit and mental health after three types of disaster. J Consult Clin Psychol 1997;65:733-739.

19. Danoff-Burg S, Revenson TA: Benefit-finding among patients with rheumatoid arthritis: Positive effects on interpersonal relationships. J Behav Med 2005;28:91-103.

20. Tran V, Wiebe DJ, Fortenberry KT, et al.: Benefit finding, affective reactions to diabetes stress, and diabetes management among early adolescents. Health Psychol 2011;30: 212-219.

21. Occhipinti S, Chambers SK, Lepore S, et al.: A longitudinal study of post-traumatic growth and psychological distress in colorectal cancer survivors. PLoS One 2015;10:e0139119.

22. Mols F, Vingerhoets AJ, Coebergh JW, et al.: Well-being, posttraumatic growth and benefit finding in long-term breast cancer survivors. Psychol Health 2009;24:583-595.

23. Thambyrajah C, Herold J, Altman K, et al.: "Cancer doesn't mean curtains": Benefit finding in patients with head and neck cancer in remission. J Pyschosoc Oncol 2010;28:666-682.
24. Martz E, Livneh H: Psychosocial adaptation to disability within the context of positive psychology: Findings from the literature. J Occup Rehabil 2016;26:4-12.

25. Parry C, Chesler MA: Thematic evidence of psychosocial thriving in childhood cancer survivors. Qual Health Res 2005; 15:1055-1073.

26. Barakat LP, Alderfer MA, Kazak AE: Posttraumatic growth in adolescent survivors of cancer and their mothers and fathers. J Pediatr Psychol 2006;31:413-419.

27. Rosenberg AR, Yi-Frazier JP, Wharton C, et al.: Contributors and inhibitors of resilience among adolescents and young adults with cancer. J Adolesc Young Adult Oncol 2014;3:185-193.

28. Hsieh HF, Shannon SE: Three approaches to qualitative content analysis. Qual Health Res 2005;15:1277-1288.

29. Phipps S, Long AM, Ogden J: Benefit finding scale for children: Preliminary findings from a childhood cancer population. J Pediatr Psychol 2007;32:1264-1271.

30. Jim HS, Pustejovsky JE, Park CL, et al.: Religion, spirituality, and physical health in cancer patients: A metaanalysis. Cancer 2015;121:3760-3768.

31. Rosenberg AR, Bona K, Wharton CM, et al.: Adolescent and young adult patient engagement and participation in survey-based research: A report from the "Resilience in Adolescents and Young Adults With Cancer" study. Pediatr Blood Cancer 2016;63:734-736.

32. Arnett JJ: Emerging adulthood. A theory of development from the late teens through the twenties. Am Psychol 2000; 55:469-480.

33. Eiland L, Romeo RD: Stress and the developing adolescent brain. Neuroscience 2013;249:162-171.

34. Galvan A, Rahdar A: The neurobiological effects of stress on adolescent decision making. Neuroscience 2013;249: 223-231.

35. Stevens MM, Dunsmore JC, Bennett DL, et al.: Adolescents living with life-threatening illnesses. In: Balk DE, Corr CA (eds): Adolescent Encounters with Death, Bereavement and Coping. New York, NY: Springer, 2009, pp. 115-140.

36. Pousset G, Bilsen J, De Wilde J, et al.: Attitudes of adolescent cancer survivors toward end-of-life decisions for minors. Pediatrics 2009;124:e1142-e1148.

37. Hinds PS, Drew D, Oakes LL, et al.: End-of-life care preferences of pediatric patients with cancer. J Clin Oncol 2005;23:9146-9154.

38. Wiener L, Zadeh S, Battles H, et al.: Allowing adolescents and young adults to plan their end-of-life care. Pediatrics 2012;130:897-905.

39. Jacobs S, Perez J, Cheng YI, et al.: Adolescent end of life preferences and congruence with their parents' preferences: Results of a survey of adolescents with cancer. Pediatr Blood Cancer 2015;62:710-714.

40. Palacios JF, Salem B, Hodge FS, et al.: Storytelling: A qualitative tool to promote health among vulnerable populations. J Transcult Nurs 2015;26:346-353.

Address correspondence to: Abby R. Rosenberg, MD, MS Cancer and Blood Disorders Center Seattle Children's Hospital 4800 Sand Point Way NE MS MB.8.501, PO Box 5371 Seattle, WA 98145-5005

E-mail: abby.rosenberg@ @eattlechildrens.org 